\title{
Anomaly matching on the Higgs branch
}

\section{Hiroyuki Shimizu, Yuji Tachikawa and Gabi Zafrir}

Kavli Institute for the Physics and Mathematics of the Universe, University of Tokyo, 5-1-5 Kashiwanoha, Kashiwa, Chiba, 277-8583 Japan

E-mail: shimizu@hep-th.phys.s.u-tokyo.ac.jp, yuji.tachikawa@ipmu.jp, gabi.zafrir@ipmu.jp

ABStRACT: We point out that we can almost always determine by the anomaly matching the full anomaly polynomial of a supersymmetric theory in $2 \mathrm{~d}, 4 \mathrm{~d}$ or $6 \mathrm{~d}$ if we assume that its Higgs branch is the one-instanton moduli space of some group $G$. This method not only provides by far the simplest method to compute the central charges of known theories of this class, e.g. $4 \mathrm{~d} E_{6,7,8}$ theories of Minahan and Nemeschansky or the 6d E-string theory, but also gives us new pieces of information about unknown theories of this class.

KEYwords: Anomalies in Field and String Theories, Supersymmetric Gauge Theory

ARXIV EPRINT: 1703.01013 


\section{Contents}

1 Introduction 1

2 Basic idea 2

3 Summary of results $\quad 4$

3.1 Six-dimensional theories 4

3.2 Four-dimensional theories 4

3.3 Two-dimensional theories 5

$\begin{array}{ll}3.4 & \text { Cases with pure gauge anomalies and/or gauge-R anomalies }\end{array}$

4 Six-dimensional theories $\quad 7$

4.1 $G$ is one of the exceptionals $\quad 7$

$4.2 G$ is $\mathrm{SU}(2) \quad 8$

$4.3 G$ is $\mathrm{SU}(3) \quad 8$

$4.4 G$ is of type $\mathrm{Sp} \quad 9$

$\begin{array}{lr}4.5 G \text { is of type SO } & 10\end{array}$

$\begin{array}{ll}4.6 G \text { is of type SU } & 10\end{array}$

$\begin{array}{lll}4.7 & \text { Global anomalies } & 11\end{array}$

5 Four-dimensional theories $\quad 11$

$5.1 G$ is of type Sp or one of the exceptionals 11

$\begin{array}{ll}5.2 G \text { is of type SO } & 12\end{array}$

$\begin{array}{ll}5.3 G \text { is of type SU } & 13\end{array}$

$\begin{array}{lll}5.4 \text { Global anomalies } & 13\end{array}$

6 Two-dimensional theories $\quad 14$

6.1 $G$ is of type Sp or one of the exceptionals 14

$\begin{array}{ll}6.2 G \text { is of type SO } & 15\end{array}$

$\begin{array}{ll}6.3 G \text { is of type SU } & 16\end{array}$

$\begin{array}{ll}\text { A Decomposition of characteristic classes } & 18\end{array}$

\section{Introduction}

Instantons of classical groups can be described in terms of the ADHM construction [1], which can in turn be realized as the Higgs branch of supersymmetric gauge theories $[2,3]$. These gauge theories arise as the worldvolume theories on perturbative $p$-branes probing perturbative $(p+4)$-branes, and the motion into the Higgs branch corresponds to the process where $p$-branes get absorbed as instantons of the gauge fields on $(p+4)$-branes. 
In string/M/F theory, there are also non-perturbative branes that host exceptional gauge groups, and if we probe them by lower-dimensional branes, we get supersymmetric theories whose Higgs branch equals to the instanton moduli spaces of exceptional groups. Among them we can count the $4 \mathrm{~d}$ theories of Minahan and Nemeschansky [4,5] for $E_{6,7,8}$ instantons and the 6d E-string theory [6, 7].

The theories obtained this way do not usually have any conventional Lagrangian descriptions, and were therefore rather difficult to study. Even their anomaly polynomials, or equivalently the conformal central charges assuming that they become superconformal in the infrared, needed to be computed first with stringy techniques $[8,9]$ and then with rather lengthy field theoretical arguments on the Coulomb branch in $4 \mathrm{~d}$ or on the tensor branch in $6 \mathrm{~d}[10-12]$.

In this paper, we point out that the anomaly matching on the Higgs branch almost always allows us to determine the full anomaly polynomial, when the theory is $6 \mathrm{~d} \mathcal{N}=(1,0)$, $4 \mathrm{~d} \mathcal{N}=2$, or $2 \mathrm{~d} \mathcal{N}=(4,0)$, and when the Higgs branch is assumed to be the one-instanton moduli space of some group $G$. This is because on the generic point of the Higgs branch the theory becomes free and the unbroken symmetry still knows the $\mathrm{SU}(2)_{R}$ symmetry at the origin.

This method provides the simplest way to compute the anomaly polynomials of $4 \mathrm{~d}$ theories of Minahan and Nemeschansky and the 6d E-string theory. But more importantly, this method gives us new pieces of information about a theory whose Higgs branch is the one-instanton moduli space of the group $G$, even when no string/M/F theory construction is known. For example, in [13], the conformal bootstrap method was used to determine the conformal central charges of the $4 \mathrm{~d}$ theory whose Higgs branch is the one-instanton moduli space of $G_{2}$ or $F_{4}$. Our method reproduces the values they obtained, and not only that, we find a strong indication that the $F_{4}$ theory does not exist because of a field theoretical inconsistency. Similarly, we will see that there cannot be any $6 \mathrm{~d} E_{6,7}$ theory.

The rest of the paper is organized as follows. In section 2 , we describe in more detail how the anomaly matching on the Higgs branch works if the Higgs branch is the oneinstanton moduli space of some group $G$. In section 3 , we summarize the results which we obtained in this paper. Then in section $4,5,6$, we study the $6 \mathrm{~d} \mathcal{N}=(1,0)$ theories, the $4 \mathrm{~d} \mathcal{N}=2$ theories, and the $2 \mathrm{~d} \mathcal{N}=(4,0)$ theories in turn. In appendix $\mathrm{A}$, we collect the formulas for characteristic classes used throughout in this paper.

\section{Basic idea}

We consider a theory with $6 \mathrm{~d} \mathcal{N}=(1,0)$ or $4 \mathrm{~d} \mathcal{N}=2$ or $2 \mathrm{~d} \mathcal{N}=(4,0)$ supersymmetry has a Higgs branch given by the one-instanton moduli space $M_{G}$ of a group $G$.

Geometric data. Let us first recall some basic information on $M_{G}$, whose detail can be found e.g. in [14] and the references therein. The quaternionic dimension of $M_{G}$ is $h^{\vee}(G)-1$. We note that for $G=\operatorname{Sp}(n)$, the one-instanton moduli space is simply $\mathbb{H}^{n} / \mathbb{Z}_{2}$, where $\mathbb{H}$ is the space of quaternions.

Furthermore, the moduli space is smooth on a generic point, and the symmetry $\mathrm{SU}(2)_{R} \times G$ acting on $M_{G}$ is broken to $\mathrm{SU}(2)_{D} \times G^{\prime}$, where $\mathrm{SU}(2)_{X} \times G^{\prime} \subset G$ is a particular 


\begin{tabular}{|c|c|c|c|l|}
\hline$G$ & $h^{\vee}$ & $G^{\prime}$ & $R^{\prime}$ & short comment \\
\hline $\mathrm{SU}(n)$ & $n$ & $\mathrm{U}(1)_{F} \times \mathrm{SU}(n-2)$ & $(\mathbf{n}-\mathbf{2})_{-n} \oplus(\overline{\mathbf{n - 2}})_{+n}$ & \\
$\mathrm{SO}(n)$ & $n-2$ & $\mathrm{SU}(2)_{F} \times \mathrm{SO}(n-4)$ & $\mathbf{2}_{F} \otimes(\mathbf{n}-\mathbf{4})$ & \\
$\mathrm{Sp}(n)$ & $n+1$ & $\mathrm{Sp}(n-1)$ & $\mathbf{2 n - 2}$ & \\
$E_{6}$ & 12 & $\mathrm{SU}(6)$ & $\mathbf{2 0}$ & 3-index antisym. \\
$E_{7}$ & 18 & $\mathrm{SO}(12)$ & $\mathbf{3 2}$ & chiral spinor. \\
$E_{8}$ & 30 & $E_{7}$ & $\mathbf{5 6}$ & \\
$F_{4}$ & 9 & $\mathrm{Sp}(3)$ & $\mathbf{1 4}$ & 3-index antisym. traceless. \\
$G_{2}$ & 4 & $\mathrm{SU}(2)_{F}$ & $\mathbf{4}$ & 3-index sym. \\
\hline
\end{tabular}

Table 1. The data. For $\mathrm{SU}(n), \mathrm{U}(1)_{F}$ is normalized so that $\mathbf{n}$ splits as $(\mathbf{n}-\mathbf{2})_{-2}$ and $\mathbf{2}_{n-2}$. For $\mathrm{SO}(n), n$ is assumed to be $\geq 5$.

subgroup described in more detail below and $\mathrm{SU}(2)_{D}$ is the diagonal subgroup of $\mathrm{SU}(2)_{R}$ and $\mathrm{SU}(2)_{X}$. The subgroup $\mathrm{SU}(2)_{X}$ is the $\mathrm{SU}(2)$ subgroup associated to the highest root of $G$ and $G^{\prime}$ is its commutant within $G$. The tangent space of $M_{G}$ at a generic point transforms under $\mathrm{SU}(2)_{X} \times G^{\prime}$ as a neutral hypermultiplet and a charged half-hypermultiplet in a representation $R$, with the rule

$$
\mathfrak{g}=\mathfrak{g}^{\prime} \oplus \mathfrak{s u}(2) \oplus R
$$

Here $R$ is always of the form of the doublet of $\mathrm{SU}(2)_{X}$ tensored with a representation $R^{\prime}$ of $G^{\prime}$. The subgroup $G^{\prime}$ and the representation $R^{\prime}$ are given in the table 1 .

Strategy of the matching. Now let us explain how the anomaly matching on the Higgs branch works. At the origin, the theory has the symmetry $\mathrm{SU}(2)_{R} \times G$ where $\mathrm{SU}(2)_{R}$ is (part of) the R-symmetry.

On a generic point of the Higgs branch, we have a free theory whose unbroken symmetry is $\mathrm{SU}(2)_{D} \times G^{\prime}$, where $\mathrm{SU}(2)_{D}$ is the diagonal subgroup of $\mathrm{SU}(2)_{R}$ and $\mathrm{SU}(2)_{X}$. The theory is a collection of $d_{H}=h^{\vee}-1$ hypermultiplets. One, identified with changing the vev, is neutral under the unbroken global symmetry. Considering that the scalars in a halfhyper are doublets of $\mathrm{SU}(2)_{R}$, this hyper should just be a half-hyper in the $\mathbf{2}$ of $\mathrm{SU}(2)_{X}$. Additionally we have the remaining $d_{H}-1$ hypers which transform as a doublet of SU $(2)_{X}$ and in some representation $R^{\prime}$ of $G^{\prime}$ given in table 1 . Since $\mathrm{SU}(2)_{X}$ and $\mathrm{SU}(2)_{R}$ are identified to be $\mathrm{SU}(2)_{D}$, this amounts to just $d_{H}-1$ free hypers in the representation $R^{\prime}$.

The anomaly of $G$ of the original theory can be found from the anomaly of $G^{\prime}$ of the free theory in the infrared, if $G^{\prime}$ is nonempty. This in turn determines the contribution of $\mathrm{SU}(2)_{X}$ to the anomaly of $\mathrm{SU}(2)_{D}$, which then fixes the anomaly of $\mathrm{SU}(2)_{R}$ of the original theory. Even if $G^{\prime}$ is empty, this still constrains the anomaly of $\mathrm{SU}(2)_{R}$ and $G$ of the original theory. Along the way, we might find that the anomaly matching cannot be satisfied, in which case we conclude that such a theory cannot exist. There are cases 
where the anomaly polynomials can be arranged to match but the global anomaly fails to match. ${ }^{1}$ We call this the global anomaly matching test.

Finally, since the one-instanton moduli space of $\operatorname{Sp}(n)$ is $\mathbb{H}^{n} / \mathbb{Z}_{2}$ as explained below, we should always be able to match the anomaly in this case by $n$ free hypermultiplets gauged by $\mathbb{Z}_{2}$, or equivalently an $\mathrm{O}(1)-\mathrm{Sp}(n)$ bifundamental gauged by $\mathrm{O}(1)$. This provides us a simple way to check the computations.

Now that the strategy has been explained, we move on to the details. We first summarize the results in the next section, and then look at the three cases in turn, in the order $6 \mathrm{~d}, 4 \mathrm{~d}$ and $2 \mathrm{~d}$.

\section{Summary of results}

In this section we summarize the results we obtain in each spacetime dimensions, postponing the computational details in the following sections. We assume that there are just free hypermultiplets on the generic point on the Higgs branch unless otherwise stated.

\subsection{Six-dimensional theories}

First we consider $6 \mathrm{~d} \mathcal{N}=(1,0)$ theories. We find that the anomaly polynomials on the Higgs branch can consistently be matched for

$$
\mathrm{SU}(2), \quad \mathrm{SU}(3), \quad \mathrm{Sp}(n), \quad E_{8}, \quad \text { and } \quad G_{2} \text {. }
$$

- In the $\mathrm{SU}(2)$ case, we cannot completely determine the anomaly at the origin; we find a three-parameter family of solutions (4.9). The result is consistent with one known example, which is just a free hypermultiplet gauged by $\mathbb{Z}_{2}$.

- In the $\mathrm{SU}(3)$ case, we can unambiguously determine the anomaly as in (4.12). But we do not know any example of $6 \mathrm{~d}$ theories with this Higgs branch.

- The $\operatorname{Sp}(n)$ case reproduces the anomaly polynomial of $n$ free hypermultiplets gauged by $\mathbb{Z}_{2}$.

- The $E_{8}$ case reproduces the anomaly of the rank-1 E-string theory.

- The $G_{2}$ case does not pass the anomaly matching test of the global anomaly, as detailed in section 4.7

\subsection{Four-dimensional theories}

Second we consider $4 \mathrm{~d} \mathcal{N}=2$ theories. We find that the anomaly polynomials on the Higgs branch can be consistently matched only for

$$
\mathrm{SU}(2), \quad \mathrm{SU}(3), \quad \mathrm{SO}(8), \quad \mathrm{Sp}(n), \quad E_{6,7,8}, \quad F_{4}, \quad \text { and } \quad G_{2} .
$$

\footnotetext{
${ }^{1}$ The mismatch of the global anomaly associated to $\pi_{d}(G)$ cannot be canceled by adding topological degrees of freedom. For details, see section 5 of [15].
} 


\begin{tabular}{|c|c|cc|cc|}
\hline$G$ & $k$ & $n_{v}$ & $n_{h}$ & $a$ & $c$ \\
\hline $\mathrm{SU}(2)$ & $x+1$ & $x$ & $x+1$ & $\frac{6 x+1}{24}$ & $\frac{3 x+1}{12}$ \\
$\mathrm{SU}(3)$ & 3 & 2 & 4 & $\frac{7}{12}$ & $\frac{2}{3}$ \\
$\mathrm{SO}(8)$ & 4 & 3 & 8 & $\frac{23}{24}$ & $\frac{7}{6}$ \\
$\mathrm{Sp}(n)$ & 1 & 0 & $n$ & $\frac{n}{24}$ & $\frac{n}{12}$ \\
$E_{6}$ & 6 & 5 & 16 & $\frac{41}{24}$ & $\frac{13}{6}$ \\
$E_{7}$ & 8 & 7 & 24 & $\frac{59}{24}$ & $\frac{19}{6}$ \\
$E_{8}$ & 12 & 11 & 40 & $\frac{95}{24}$ & $\frac{31}{6}$ \\
\hline$F_{4}$ & 5 & 4 & 12 & $\frac{4}{3}$ & $\frac{5}{3}$ \\
$G_{2}$ & $\frac{10}{3}$ & $\frac{7}{3}$ & $\frac{16}{3}$ & $\frac{17}{24}$ & $\frac{5}{6}$ \\
\hline
\end{tabular}

Table 2. The cases compatible with conformal symmetry in four dimensions. For SU(2) the parameter $x$ can not be fixed by our method. Those except $F_{4}$ and $G_{2}$ are known to exist. The $F_{4}$ case suffers from the mismatch of the global anomaly.

The data is summarized in table 2, using the standard notations. The list of the groups we found here is equal to the list of group compatible with the one-instanton moduli space as Higgs branches, determined using the conformal bootstrap in $[13,16] .{ }^{2}$ Note however that the $F_{4}$ case does not pass the global anomaly matching test, as will be detailed in section 5.4.

\subsection{Two-dimensional theories}

Third, we consider $2 \mathrm{~d} \mathcal{N}=(4,0)$ theories. In two dimensions, the scalars always fluctuate all over the moduli space, and the continuous symmetry never breaks. Therefore, it is not technically correct to speak of the theory at the origin of the moduli space and compare the anomaly computed at the generic point. Rather, what we do is to match the anomaly polynomial as calculated using a semi-classical analysis at the generic point using the unbroken symmetry at that point, with the anomaly polynomial written in terms of the full symmetry.

We find that the anomaly polynomials on the Higgs branch can consistently be matched only for

$$
\mathrm{SU}(2), \quad \mathrm{SU}(3), \quad \mathrm{SO}(8), \quad \mathrm{Sp}(n), \quad E_{6,7,8}, \quad F_{4}, \quad \text { and } \quad G_{2} .
$$

The data is summarized in table 3 , where $n_{v}, d_{H}, k_{G}$ are the coefficients in the anomaly polynomial expanded as follows:

$$
I_{4}^{\text {full }}=-n_{v} c_{2}(R)+d_{H} c_{2}(I)+\frac{2 d_{H}-n_{f}}{24} p_{1}(T)+\frac{k_{G}}{4} \operatorname{Tr}\left(F_{G}^{2}\right)
$$

\footnotetext{
${ }^{2}$ Note that in table 4 of [13] $\mathrm{Sp}(n)$ is missing. This is because the authors of [13] assumed that the theory is interacting in constructing their table 4 . The authors thank L. Rastelli for the clarifications.
} 


\begin{tabular}{|c|c|c|c|c|}
\hline$G$ & $n$ & $n_{v}$ & $d_{H}$ & $k_{G}$ \\
\hline $\mathrm{SU}(2)$ & & $x-1$ & 1 & $x$ \\
$\mathrm{Sp}(n)$ & & 0 & $n$ & 1 \\
\hline $\mathrm{SU}(3)$ & 3 & 2 & 2 & 3 \\
$\mathrm{SO}(8)$ & 4 & 3 & 5 & 4 \\
$F_{4}$ & 5 & 4 & 8 & 5 \\
$E_{6}$ & 6 & 5 & 11 & 6 \\
$E_{7}$ & 8 & 7 & 17 & 8 \\
$E_{8}$ & 12 & 11 & 29 & 12 \\
\hline$G_{2}$ & & $\frac{7}{3}$ & 3 & $\frac{10}{3}$ \\
\hline
\end{tabular}

Table 3. The cases without Fermi multiplets in two dimensions. We explicitly show the value of self-Dirac-Zwazinger paring as $n$ when the theory is realized on a single string in minimal $6 \mathrm{~d}$ $\mathcal{N}=(1,0)$ theories.

where $\mathrm{SU}(2)_{R}$ and $\mathrm{SU}(2)_{I}$ are the R-symmetries. Note that there is no global anomaly test in $2 \mathrm{~d}$.

- The $\operatorname{Sp}(n)$ case gives us the anomaly of $n$ free hypermultiplets gauged by $\mathbb{Z}_{2}$.

- The $\mathrm{SU}(3), \mathrm{SO}(8), E_{6,7,8}, F_{4}$ cases reproduce the anomaly on a single string in $6 \mathrm{~d}$ minimal gauge theories $[17,18]$. These theories are also realized as compactifications of the corresponding $4 \mathrm{~d} \mathcal{N}=2$ theories on $S^{2}$ [19].

- For the $\mathrm{SU}(2)$ case, we cannot completely determine the anomalies.

- For the $G_{2}$ case, we do not know any example of 2 d theories with these values of anomalies.

In two dimensions, we can slightly generalize the situation by allowing the massless Fermi multiplets on the Higgs branch. The inclusion of Fermi multiplets opens the possibility of matching the anomaly even for larger $\mathrm{SU}(n)$ and $\mathrm{SO}(n)$ groups. We analyze several examples with relatively simple Fermi multiplet spectrum and reproduce the anomaly of a single string in $6 \mathrm{~d}$ non-anomalous gauge theory with various matter hypermultiplets, as we will show in detail in section 6 .

\subsection{Cases with pure gauge anomalies and/or gauge- $\mathrm{R}$ anomalies}

In two, four and six dimensions, we find that for larger $\mathrm{SU}(n)$ and $\mathrm{SO}(n)$ groups, we cannot consistently match the anomaly polynomial. Still, if we ignore the matching of the terms associated to $\mathrm{U}(1)_{F}$ and $\mathrm{SU}(2)_{F}$ (which are subgroups of unbroken flavor symmetries as given in table 1), we find that our method somehow reproduces the values of anomalies which one would naively associate to the ADHM gauge theories realizing the one-instanton moduli spaces of these groups. 
In four dimension, these theories are infrared free, and have a mixed gauge-gauge- $\mathrm{R}$ anomaly. Moreover, for SO(odd), the gauge group has the global anomaly. In two and six dimensions, these theories have a gauge anomaly.

We do not understand why the anomaly matching partially works for these cases. It seems that the anomalies involving the gauge fields plays the role. We hope to come back to study this case further.

\section{Six-dimensional theories}

In this section we perform the analysis for $6 \mathrm{~d} \mathcal{N}=(1,0)$ theories.

\section{1 $G$ is one of the exceptionals}

First, let us specialize to the exceptional groups. Since there is no independent quartic Casimir for exceptional groups, the anomaly polynomial at the origin can be written as

$$
\begin{aligned}
I_{8}^{\text {origin }}= & \alpha c_{2}(R)^{2}+\beta c_{2}(R) p_{1}(T)+\gamma p_{1}(T)^{2}+\delta p_{2}(T) \\
& +\frac{1}{4} \operatorname{Tr} F_{G}^{2}\left(\frac{\kappa}{4} \operatorname{Tr} F_{G}^{2}+\lambda c_{2}(R)+\mu p_{1}(T)\right),
\end{aligned}
$$

where $c_{2}(R)$ is the second Chern class of the $\mathrm{SU}(2)$ R-symmetry bundle and $p_{1}(T), p_{2}(T)$ are the first and second Pontryagin classes of the tangent bundle respectively. We have also introduced the unknown coefficients $\alpha, \beta, \gamma, \delta, \kappa, \lambda, \mu$ to be determined below. On the generic point of the Higgs branch, using (A.2) we see that the anomaly polynomial (4.1) becomes

$$
\begin{aligned}
I_{8}^{\text {generic }}= & (\alpha+\kappa+\lambda) c_{2}(D)^{2}+(\beta+\mu) c_{2}(D) p_{1}(T)+\gamma p_{1}(T)^{2}+\delta p_{2}(T) \\
& +\frac{m}{4} \operatorname{Tr} F_{G^{\prime}}^{2}\left(\frac{m \kappa}{4} \operatorname{Tr} F_{G^{\prime}}^{2}+(2 \kappa+\lambda) c_{2}(D)+\mu p_{1}(T)\right) .
\end{aligned}
$$

On the other hand, the anomaly of free hypers is given as

$$
\begin{aligned}
I_{8}^{\text {hypers }}= & \frac{1}{24} c_{2}(D)^{2}+\frac{1}{48} c_{2}(D) p_{1}(T)+\frac{7\left(2+d_{R^{\prime}}\right)}{11520} p_{1}(T)^{2}-\frac{2+d_{R^{\prime}}}{2880} p_{2}(T) \\
& +\frac{1}{48} \operatorname{tr}_{R^{\prime}} F_{G^{\prime}}^{4}+\frac{T^{G^{\prime}}\left(R^{\prime}\right)}{96} \operatorname{Tr} F_{G^{\prime}}^{2} p_{1}(T) .
\end{aligned}
$$

In order to match (4.2) and (4.3), there should also be no independent quartic Casimir invariant for $G^{\prime}$. This already excludes $G=E_{6}, E_{7}, F_{4}$ and the remaining possibilities are $G=G_{2}, E_{8}$.

When $\boldsymbol{G}=\boldsymbol{E}_{\mathbf{8}}$. Since $\operatorname{tr}_{\mathbf{5 6}} F_{E_{7}}^{4}=\frac{3}{2}\left(\operatorname{Tr} F_{E_{7}}^{2}\right)^{2}$ and $T^{E_{7}}(\mathbf{5 6})=6$, the anomaly (4.3) becomes

$$
\begin{aligned}
I_{8}^{\text {hyper }}= & \frac{1}{24} c_{2}(D)^{2}+\frac{1}{48} c_{2}(D) p_{1}(T)+\frac{203}{5760} p_{1}(T)^{2}-\frac{29}{1440} p_{2}(T) \\
& +\frac{1}{32}\left(\operatorname{Tr} F_{G^{\prime}}^{2}\right)^{2}+\frac{1}{16} \operatorname{Tr} F_{G^{\prime}}^{2} p_{1}(T) .
\end{aligned}
$$


Comparing (4.2) and (4.4), we can solve as

$\alpha=\frac{13}{24}, \quad \beta=-\frac{11}{48}, \quad \gamma=\frac{203}{5760}, \quad \delta=-\frac{29}{1440}, \quad \kappa=\frac{1}{2}, \quad \lambda=-1, \quad \mu=\frac{1}{4}$

which coincides with the anomaly of rank-1 E-string theory determined in [9].

When $\boldsymbol{G}=\boldsymbol{G}_{\mathbf{2}}$. Using $\operatorname{tr}_{\mathbf{4}} F_{\mathrm{SU}(2)}^{4}=\frac{41}{4}\left(\operatorname{Tr} F_{\mathrm{SU}(2)}^{2}\right)^{2}$ and $T^{\mathrm{SU}(2)}(\mathbf{4})=5$, the anomaly (4.3) becomes

$$
\begin{aligned}
I_{8}^{\text {hypers }}= & \frac{1}{24} c_{2}(D)^{2}+\frac{1}{48} c_{2}(D) p_{1}(T)+\frac{7}{1920} p_{1}(T)^{2}-\frac{1}{480} p_{2}(T) \\
& +\frac{41}{192}\left(\operatorname{Tr} F_{\mathrm{SU}(2)}^{2}\right)^{2}+\frac{5}{96} \operatorname{Tr} F_{\mathrm{SU}(2)}^{2} p_{1}(T) .
\end{aligned}
$$

Comparing (4.2) and (4.6), we can solve finding

$$
\alpha=\frac{91}{216}, \quad \beta=-\frac{7}{144}, \quad \gamma=\frac{7}{1920}, \quad \delta=-\frac{1}{480}, \quad \kappa=\frac{41}{108}, \quad \lambda=-\frac{41}{54}, \quad \mu=\frac{5}{72} .
$$

\section{2 $G$ is $\mathrm{SU}(2)$}

Let us consider the $\mathrm{SU}(2)$ case, which is quite exceptional. In this case, the anomaly of the hypermultiplet is just

$$
I_{8}^{\text {hypers }}=\frac{c_{2}(D)^{2}}{24}+\frac{c_{2}(D) p_{1}(T)}{48}+\frac{7 p_{1}(T)^{2}-4 p_{2}(T)}{5760} .
$$

The anomaly polynomial of the SCFT is still of the form (4.1) since there is no independent quartic Casimir. On a generic point of the Higgs branch $\mathrm{SU}(2)_{G}$ and $\mathrm{SU}(2)_{R}$ are identified which is implemented by (A.9). Matching the resulting anomaly polynomial with (4.8), we find:

$$
\begin{aligned}
\alpha+\kappa+\lambda & =\frac{1}{24}, \\
\beta+\mu & =\frac{1}{48}, \\
\gamma & =\frac{7}{5760}, \quad \delta=\frac{1}{1440} .
\end{aligned}
$$

In this case we cannot determine the anomaly polynomial completely. The known $6 \mathrm{~d}$ theory that have the Higgs branch $M_{\mathrm{SU}(2)}$ is the $\mathrm{O}(1) \times \mathrm{SU}(2)$ free hyper. The anomaly polynomial of this theory is consistent with (4.9) with $\alpha=\beta=\lambda=0$.

\section{3 $G$ is $\mathrm{SU}(3)$}

The case of $\mathrm{SU}(3)$ is also exceptional since the fourth Casimir of $\mathrm{SU}(3)$ is zero and we can take the SCFT anomaly polynomial to be of the form (4.1). Substituting the decomposition (A.10) to (4.1), we obtain

$$
\begin{aligned}
I_{8}^{\text {generic }}= & (\alpha+\kappa+\lambda) c_{2}(D)^{2}+(\beta+\mu) c_{2}(D) p_{1}(T)+\gamma p_{1}(T)^{2}+\delta p_{2}(T) \\
& -3 c_{1}\left(\mathrm{U}(1)_{F}\right)^{2}\left((\lambda+2 \kappa) c_{2}(D)+\mu p_{1}(T)-3 \kappa c_{1}\left(\mathrm{U}(1)_{F}\right)^{2}\right)
\end{aligned}
$$


In turn the anomaly polynomial of the free hypers is given by:

$$
\begin{aligned}
I_{8}^{\text {hypers }}=\frac{c_{2}(D)^{2}}{24}+ & \frac{c_{2}(D) p_{1}(T)}{48} \\
& -\frac{3 c_{1}\left(\mathrm{U}(1)_{F}\right)^{2} p_{1}(T)}{16}+\frac{27 c_{1}\left(\mathrm{U}(1)_{F}\right)^{4}}{8}+2 \frac{7 p_{1}(T)^{2}-4 p_{2}(T)}{5760} .
\end{aligned}
$$

Comparing the two we find a unique solution:

$$
\begin{aligned}
& \alpha=\frac{5}{12}, \quad \beta=-\frac{1}{24}, \\
& \gamma=\frac{7}{2880}, \quad \delta=-\frac{1}{720}, \\
& \kappa=\frac{3}{8}, \quad \lambda=-\frac{3}{4}, \quad \mu=\frac{1}{16} .
\end{aligned}
$$

To our knowledge, a 6d SCFT with this Higgs branch is not known.

\section{4 $G$ is of type Sp}

In this case, the anomaly of the free hypers is given by

$$
I_{8}^{\text {hypers }}=\frac{1}{48}\left(\operatorname{tr}_{\text {fund }} F_{\mathrm{Sp}(n-1)}^{4}+2 c_{2}(D)^{2}\right)+\frac{\left(2 c_{2}(D)+\operatorname{tr}_{\text {fund }} F_{\mathrm{Sp}(n-1)}^{2}\right) p_{1}(T)}{96}+n \frac{7 p_{1}(T)^{2}-4 p_{2}(T)}{5760} .
$$

Since the purely gravitational part of the anomaly can be reproduced from that of the free hypers, we focus on the R-symmetry and the flavor symmetry part written as

$$
I_{8}^{\text {origin }}=\alpha c_{2}(R)^{2}+\beta c_{2}(R) p_{1}(T)+x \operatorname{tr}_{\text {fund }} F_{\mathrm{Sp}(n)}^{4}+y\left(\operatorname{tr}_{\text {fund }} F_{\mathrm{Sp}(n)}^{2}\right)^{2}+\operatorname{tr}_{\text {fund }} F_{\mathrm{Sp}(n)}^{2}\left(\kappa c_{2}(R)+\lambda p_{1}(T)\right) .
$$

Decomposing the characteristic classes for $\operatorname{Sp}(n)$ to those for $\operatorname{Sp}(n-1)$ using (A.3) and (A.4), we find that the anomaly becomes:

$$
\begin{aligned}
I_{8}^{\text {generic }}=(\alpha+2 x+ & 4 y+2 \kappa) c_{2}(D)^{2}+(\beta+2 \lambda) c_{2}(D) p_{1}(T) \\
& +x \operatorname{tr}_{\text {fund }} F_{\mathrm{Sp}(n-1)}^{4}+4 y c_{2}(D) \operatorname{tr}_{\text {fund }} F_{\mathrm{Sp}(n-1)}^{2} \\
& +y\left(\operatorname{tr}_{\text {fund }} F_{\mathrm{Sp}(n-1)}^{2}\right)^{2}+\operatorname{tr}_{\text {fund }} F_{\mathrm{Sp}(n-1)}^{2}\left(\kappa c_{2}(D)+\lambda p_{1}(T)\right) .
\end{aligned}
$$

Comparing (4.13) and (4.15), we find

$$
\alpha=0, \quad \beta=0, \quad x=\frac{1}{48}, \quad y=0, \quad \kappa=0, \quad \lambda=\frac{1}{96},
$$

which coincides with the anomaly of $\mathrm{O}(1) \times \mathrm{Sp}(n)$ half-hyper when we include the purely gravitational part. This SCFT is the ADHM gauge theory for $\operatorname{Sp}(n)$. 


\section{5 $G$ is of type SO}

In this case, the anomaly of the hypermultiplet is given by

$$
\begin{aligned}
I_{8}^{\text {hypers }}= & \frac{(n-4) \operatorname{tr}_{\text {fund }} F_{F}^{4}+6 \operatorname{tr}_{\text {fund }} F_{F}^{2} \operatorname{tr}_{\text {fund }} F_{\mathrm{SO}(n-4)}^{2}+2 \operatorname{tr}_{\text {fund }} F_{\mathrm{SO}(n-4)}^{4}+2 c_{2}(D)^{2}}{48} \\
& +\frac{\left((n-4) \operatorname{tr}_{\text {fund }} F_{F}^{2}+2 \operatorname{tr}_{\text {fund }} F_{\mathrm{SO}(n-4)}^{2}+2 c_{2}(D)\right) p_{1}(T)}{96}+(n-3) \frac{7 p_{1}(T)^{2}-4 p_{2}(T)}{5760} .
\end{aligned}
$$

Since the purely gravitational part reproduces the anomaly at the origin, we concentrate on the part involving the R-symmetry and the flavor symmetry. We write the anomaly at the origin as

$$
I_{8}^{\text {origin }}=\alpha c_{2}(R)^{2}+\beta c_{2}(R) p_{1}(T)+x \operatorname{tr}_{\text {fund }} F_{\mathrm{SO}(n)}^{4}+y\left(\operatorname{tr}_{\text {fund }} F_{\mathrm{SO}(n)}^{2}\right)^{2}+\operatorname{tr}_{\text {fund }} F_{\mathrm{SO}(n)}^{2}\left(\kappa c_{2}(R)+\lambda p_{1}(T)\right) .
$$

We can use equations (A.5) and (A.6) to get:

$$
\begin{aligned}
I_{8}^{\text {generic }}= & (\alpha+4 x+16 y+4 \kappa) c_{2}(R)^{2}+(\beta+4 \lambda) c_{2}(D) p_{1}(T)+(x+4 y)\left(\operatorname{tr}_{\text {fund }} F_{F}^{2}\right)^{2} \\
& +(12 x+16 y+2 \kappa) c_{2}(D) \operatorname{tr}_{\text {fund }} F_{F}^{2}+x \operatorname{tr}_{\text {fund }} F_{\mathrm{SO}(n-4)}^{4}+(16 y+\kappa) c_{2}(D) \operatorname{tr}_{\text {fund }} F_{\mathrm{SO}(n-4)}^{2} \\
& +y\left(\operatorname{tr}_{\text {fund }} F_{\mathrm{SO}(n-4)}^{2}\right)^{2}+4 y \operatorname{tr}_{\text {fund }} F_{F}^{2} \operatorname{tr}_{\text {fund }} F_{\mathrm{SO}(n-4)}^{2}+\lambda p_{1}(T)\left(\operatorname{tr}_{\text {fund }} F_{\mathrm{SO}(n-4)}^{2}+2 \operatorname{tr}_{\text {fund }} F_{F}^{2}\right) .
\end{aligned}
$$

What we have to do is to match (4.17) and (4.20) and solve for $\alpha, \beta, x, y, \kappa, \lambda$. We see that the $\mathrm{SU}(2)_{F}$ independent terms can be matched by setting $\alpha=-\frac{1}{8}, \beta=-\frac{1}{16}, x=$ $\frac{1}{24}, \lambda=\frac{1}{48}, y=\kappa=0$. These are the values one get for an $\mathrm{SU}(2)$ gauge theory with $n$ half-hypermultiplets though it is anomalous in $6 \mathrm{~d}$. However it is not possible to much the remaining $\mathrm{SU}(2)_{F}$ dependent terms so there is no solution in this case.

\section{6 $G$ is of type SU}

We only need to consider $n \geq 4$. Then, the anomaly of the hypermultiplets is given by

$$
\begin{aligned}
I_{8}^{\text {hypers }}= & \frac{c_{2}(D)^{2}}{24}+\frac{c_{2}(D) p_{1}(T)}{48}-\frac{n^{2}(n-2) c_{1}\left(\mathrm{U}(1)_{F}\right)^{2} p_{1}(T)}{48}+\frac{\operatorname{tr}_{\text {fund }} F_{\mathrm{SU}(n-2)}^{2} p_{1}(T)}{48} \\
& -\frac{n^{2} c_{1}\left(\mathrm{U}(1)_{F}\right)^{2} \operatorname{tr}_{\text {fund }} F_{\mathrm{SU}(n-2)}^{2}}{4}+\frac{n^{4}(n-2) c_{1}\left(\mathrm{U}(1)_{F}\right)^{4}}{24}+\frac{\operatorname{tr}_{\text {fund }} F_{\mathrm{SU}(n-2)}^{4}}{24} \\
& -\frac{n c_{1}\left(\mathrm{U}(1)_{F}\right) \operatorname{tr}_{\text {fund }} F_{\mathrm{SU}(n-2)}^{3}}{6}+(n-1) \frac{7 p_{1}(T)^{2}-4 p_{2}(T)}{5760} .
\end{aligned}
$$

We take the flavor and R-symmetry part of the anomaly to be given by (4.14) with the replacement $\mathrm{Sp}(n) \rightarrow \mathrm{SU}(n)$. Decomposing the characteristic classes of $\mathrm{SU}(n)$ into their $\mathrm{U}(1)_{F} \times \mathrm{SU}(n-2)$ counterparts using (A.7) and (A.8), we find:

$$
\begin{aligned}
I_{8}^{\text {generic }}= & (\alpha+2 x+4 y+2 \kappa) c_{2}(D)^{2}+(\beta+2 \lambda) c_{2}(D) p_{1}(T)+x \operatorname{tr}_{\text {fund }} F_{\mathrm{SU}(n-2)}^{4}+y\left(\operatorname{tr}_{\text {fund }} F_{\mathrm{SU}(n-2)}^{2}\right)^{2} \\
& -8 x c_{1}\left(\mathrm{U}(1)_{F}\right) \operatorname{tr}_{\text {fund }} F_{\mathrm{SU}(n-2)}^{3}-4(6 x+n(n-2) y) c_{1}\left(\mathrm{U}(1)_{F}\right)^{2} \operatorname{tr}_{\text {fund }} F_{\mathrm{SU}(n-2)}^{2} \\
& -2(n-2)(n \kappa+4 y n+6(n-2) x) c_{1}\left(\mathrm{U}(1)_{F}\right)^{2} c_{2}(D)+2 n(n-2)\left(x\left(n^{2}-6 n+12\right)\right. \\
& +2 y n(n-2)) c_{1}\left(\mathrm{U}(1)_{F}\right)^{4}+(\kappa+4 y) c_{2}(D) \operatorname{tr}_{\text {fund }} F_{\mathrm{SU}(n-2)}^{2} \\
& +\lambda \operatorname{tr}_{\text {fund }} F_{\mathrm{SU}(n-2)}^{2} p_{1}(T)-4 n(n-2) \lambda c_{1}\left(\mathrm{U}(1)_{F}\right)^{2} p_{1}(T) .
\end{aligned}
$$


Matching equations (4.20) and (4.21) we see that the $\mathrm{U}(1)_{F}$ independent terms can be matched by setting $\alpha=-x=-\frac{1}{24}, \beta=-\lambda=-\frac{1}{48}, y=\kappa=0$. These are the values one get for a $\mathrm{U}(1)$ gauge theory with $n$ hypermultiplets though it is anomalous in 6 d. The $\mathrm{U}(1)_{F}$ dependent terms only match if $n=2$ for which this analysis does not apply. Therefore we conclude that there is no solution in this case.

\subsection{Global anomalies}

Finally we consider anomalies under large gauge transformations. These exist only for groups with $\pi_{6}(G) \neq 0$ which are only $\mathrm{SU}(2), \mathrm{SU}(3)$ and $G_{2}$ for which $\pi_{6}(\mathrm{SU}(2))=$ $\mathbb{Z}_{12}, \pi_{6}(\mathrm{SU}(3))=\mathbb{Z}_{6}$ and $\pi_{6}\left(G_{2}\right)=\mathbb{Z}_{3}$. These anomalies are mapped to one another under the embedding of $\mathrm{SU}(2) \rightarrow \mathrm{SU}(3) \rightarrow G_{2}$. When embedded in groups with an independent fourth Casimir, the global anomaly can match the standard square anomaly.

A hyper in the $\mathbf{7}$ of $G_{2}$, one in the $\mathbf{3}$ of SU(3), and a half-hyper in the $\mathbf{2}$ of SU(2) both contribute to the anomaly as the generator of $\pi_{6}(G)$ for their respective groups [20]. Under the above mapping the $\mathbf{7}$ of $G_{2}$ goes to the $\mathbf{3}+\overline{\mathbf{3}}$ of SU(3) and further to the $2 \times \mathbf{2}+$ singlets of $\mathrm{SU}(2)$. Therefore the anomaly is consistently mapped across the groups.

The only non-excluded cases where the anomaly might be relevant are $\mathrm{SU}(2), \mathrm{SU}(3)$ and $G_{2}$. For SU(2) and SU(3) the anomaly doesn't exist on the Higgs branch which implies that the anomaly vanishes in the SCFT. The situation for $G_{2}$ is more involved as it is broken to $\mathrm{SU}(2)$ on the Higgs branch where both groups have the discrete anomaly.

Let's consider the 7 of $G_{2}$. Under the $\mathrm{SU}(2)_{1} \times \mathrm{SU}(2)_{2}$ subgroup of $G_{2}$, it decomposes as: $\mathbf{7} \rightarrow(\mathbf{2}, \mathbf{2}) \oplus(\mathbf{1}, \mathbf{3})$. As the anomaly must be preserved, and using the fact that the 3 of SU(2) contribute to the anomaly like 8 half-hyper doublets [20], we determine that $\mathrm{SU}(2)_{1}$ has the same anomaly as $G_{2}$ while $\mathrm{SU}(2)_{2}$ is non-anomalous. Therefore $\mathrm{SU}(2)_{2}$, which is the remaining global symmetry on a generic point on the Higgs branch, must be non-anomalous.

However on a generic point on the Higgs branch we have an half-hyper in the 4 of $\mathrm{SU}(2)_{2}$ which does contribute to the anomaly. This can be readily seen by decomposing the 14 of $G_{2}$ under the $\mathrm{SU}(2)_{1} \times \mathrm{SU}(2)_{2}$ subgroup. Thus it is apparent that we cannot match the $\mathrm{SU}(2)$ anomaly with the anomaly of $G_{2}$. Therefore the $G_{2}$ theory is inconsistent, since global anomaly associated to $\pi_{6}(G)$ cannot be canceled by adding topological degrees of freedom, as argued in e.g. section 5 of [15].

\section{Four-dimensional theories}

In this section we implement the strategy given in section 2 for $4 \mathrm{~d} \mathcal{N}=2$ theories. We perform the analysis assuming that the theories in question are superconformal. The analysis is slightly different depending on whether $G$ is a group of type SU, SO or Sp and the exceptional groups. We next discuss each in turn.

\section{1 $G$ is of type Sp or one of the exceptionals}

When the group is of type Sp or the exceptionals then the symmetry $G^{\prime}$ is a simple group. We take the anomaly polynomial of the theory to be:

$$
I_{6}^{\text {origin }}=-\frac{d_{H}}{3} c_{1}(R)^{3}+\frac{d_{H}}{12} p_{1}(T) c_{1}(R)-n_{v} c_{1}(R) c_{2}(R)+\frac{k_{G}}{4} c_{1}(R) \operatorname{Tr} F_{G}^{2}
$$




\begin{tabular}{|c|c|c|c|c|c|c|c|c|}
\hline$G$ & $E_{7}$ & $\mathrm{SO}(12)$ & $\mathrm{SU}(6)$ & $\mathrm{Sp}(3)$ & $\mathrm{SU}(2)$ & $\mathrm{Sp}(n)$ & $\mathrm{SO}(n)$ & $\mathrm{SU}(n)$ \\
\hline$R$ & $\mathbf{5 6}$ & $\mathbf{3 2}$ & $\mathbf{2 0}$ & $\mathbf{1 4}^{\prime}$ & $\mathbf{4}$ & $\mathbf{2 n}$ & $\mathbf{n}$ & $\mathbf{n}$ \\
$T^{G}(R)$ & 6 & 4 & 3 & $\frac{5}{2}$ & 5 & $\frac{1}{2}$ & 1 & $\frac{1}{2}$ \\
\hline
\end{tabular}

Table 4. The values of $T^{G}(R)$ for various representations.

where $c_{1}(R)$ is the first Chern class of the $\mathcal{N}=2 \mathrm{U}(1)$ R-symmetry bundle. The form of the anomaly polynomial is dictated by $\mathcal{N}=2$ SUSY [21]. The constants $d_{H}$ and $n_{v}$ are related to the central charges $a, c$ through: $d_{H}=24(c-a), n_{v}=4(2 a-c)$. The constant $k_{G}$ is the central charge of the flavor symmetry $G$.

The anomaly polynomial of the free hypers is:

$$
I_{6}^{\text {hypers }}=-\frac{2+d_{R^{\prime}}}{6} c_{1}(R)^{3}+\frac{2+d_{R^{\prime}}}{24} p_{1}(T) c_{1}(R)+c_{1}(R) c_{2}(D)+\frac{T^{G^{\prime}}\left(R^{\prime}\right)}{2} c_{1}(R) \operatorname{Tr} F_{G^{\prime}}^{2}
$$

Next we need to decompose the $G$-characteristic classes to the $\mathrm{SU}(2)_{D} \times G^{\prime}$ ones, where the relation is given in (A.2). By matching (5.1) and (5.2) we find:

$$
d_{H}=\frac{d_{R^{\prime}}+2}{2}, \quad n_{v}=\frac{2 T^{G^{\prime}}\left(R^{\prime}\right)}{m}-1, \quad k_{G}=\frac{2 T^{G^{\prime}}\left(R^{\prime}\right)}{m} .
$$

The values of the Dynkin index are in table 4. The complete results are summarized in table 2 .

For $\operatorname{Sp}(n)$ these are just the values of $n$ free hypers. The SCFT consisting of an $\mathrm{O}(1) \times \operatorname{Sp}(n)$ half-hyper indeed has this space as its Higgs branch.

\section{2 $G$ is of type SO}

In this case the group $G^{\prime}$ is $\mathrm{SU}(2)_{F} \times \mathrm{SO}(n-4)$ which is a semi-simple group. We again take (5.1) as the anomaly polynomial of the SCFT and decompose the $\mathrm{SO}(n)$ characteristic classes by (A.5), but now the half-hypers contribute:

$$
\begin{aligned}
I_{6}^{\text {hypers }}=-\frac{N-3}{3} c_{1}(R)^{3}+\frac{n-3}{12} & p_{1}(T) c_{1}(R)+c_{1}(R) c_{2}(D) \\
& +c_{1}(R) \operatorname{Tr} F_{\mathrm{SO}(n-4)}^{2}+\frac{(n-4)}{4} c_{1}(R) \operatorname{Tr} F_{\mathrm{SU}(2)_{F}}^{2} .
\end{aligned}
$$

Next we can proceed to match corresponding terms. Ignoring $\mathrm{SU}(2)_{F}$ terms we found that:

$$
d_{H}=n-3, \quad n_{v}=3, \quad k_{\mathrm{SO}(n)}=4 .
$$

Interestingly these are exactly the values for an $\mathrm{SU}(2)$ gauge theory with $n$ half-hypers which classically has this space as its Higgs branch. This is despite the fact that this theory has a global gauge anomaly for $n$ odd and even for $n$ even is not an SCFT unless $n=8$.

Finally we need to match the last $\mathrm{SU}(2)_{F}$ dependent term. This leads to the constraint $n-4=k_{\mathrm{SO}(n)}$ which is only obeyed if $n=8$. 


\section{3 $G$ is of type SU}

In this case the group $G^{\prime}$ is $\mathrm{U}(1)_{F} \times \mathrm{SU}(n-2)$. We again take (5.1) as the anomaly polynomial of the SCFT, but now the half-hypers contribute:

$$
\begin{aligned}
I_{6}^{\text {hypers }}=-\frac{n-1}{3} c_{1}(R)^{3}+\frac{n-1}{12} & p_{1}(T) c_{1}(R)+c_{1}(R) c_{2}(D) \\
& +\frac{1}{2} c_{1}(R) \operatorname{Tr} F_{\mathrm{SU}(n-2)}^{2}-n^{2}(n-2) c_{1}(R) c_{1}\left(\mathrm{U}(1)_{F}\right)^{2} .
\end{aligned}
$$

When $\boldsymbol{n}>$ 3. Assuming $n>3$ and ignoring $\mathrm{U}(1)_{F}$ terms we find that:

$$
d_{H}=n-1, \quad n_{v}=1, \quad k_{\mathrm{SU}(n)}=2 .
$$

Interestingly these are exactly the values for a $\mathrm{U}(1)$ gauge theory with $n$ hypers which classically has this space as its Higgs branch. This is despite the fact that this theory is not an SCFT. Indeed, to match the last $\mathrm{U}(1)_{F}$ dependent term, we need the constraint $n^{2}(n-2)=2 n(n-2)$ which has the solution $n=2$. This is incompatible with $n>3$.

When $\boldsymbol{n}=3$. For $n=3$, we now only have $\mathrm{U}(1)_{F}$ and so $\operatorname{Tr}\left(F_{\mathrm{SU}(n-2)}^{2}\right)$ vanishes. Matching terms we find:

$$
d_{H}=2, \quad n_{v}=2, \quad k_{G}=3 .
$$

These are precisely the values of the $\mathrm{AD} \mathrm{SU}(3)$ theory.

When $\boldsymbol{n}=\mathbf{2}$. For $\mathrm{SU}(2)$ we have only $\mathrm{SU}(2)_{D}$ as a remaining global symmetry and so we only get the constraints:

$$
d_{H}=1, \quad n_{v}=k_{\mathrm{SU}(2)}-1 .
$$

These are obeyed for both the $\mathrm{O}(1) \times \mathrm{SU}(2)$ half-hyper and the $\mathrm{SU}(2)$ AD theory, which are the SCFTs known to have $M_{\mathrm{SU}(2)}$ as their Higgs branch. ${ }^{3}$ Additionally it is obeyed for a $\mathrm{U}(1)$ gauge theory with two charge +1 hypermultiplets, even though it is not an SCFT.

\subsection{Global anomalies}

So far we have used local anomalies to constraint properties of $4 \mathrm{~d} \mathcal{N}=2$ theories that have the one-instanton moduli space $M_{G}$ as their Higgs branch. We can put one additional constraint using anomalies under large gauge transformation of [23]. These exist only for groups with $\pi_{4}(G) \neq 0$, which are only Sp groups for which $\pi_{4}(\operatorname{Sp}(n))=\mathbb{Z}_{2}$. When Sp group is embedded in Sp group, the global anomaly should match the global anomaly. When Sp group is embedded in SU group, the global anomaly can match the standard triangle anomaly [24].

\footnotetext{
${ }^{3}$ Other known examples of the SCFT whose Higgs branch is $M_{\mathrm{SU}(2)}=\mathbb{C}^{2} / \mathbb{Z}^{2}$ include the superconformal point of $\mathrm{SO}(4 k+2) \mathrm{SYM}[22]$. However, these theories do not fit within the class of theories considered in this paper. At the generic point on the Higgs branch, the spectrum we obtain is not free hypermultplets but the interacting SCFT without Higgs branch, i.e. the superconformal fixed point of SU(2k-1) SYM. This can be readily seen by the class $\mathrm{S}$ description of the SCFTs.
} 
In our case this implies a non-trivial constraint only for $G=\operatorname{Sp}(n), F_{4}, G_{2}$. In the first case, $G=\operatorname{Sp}(n)$, the unbroken group on the Higgs branch is $\operatorname{Sp}(n-1)$, and as the matter content is a single fundamental half-hyper, it suffers from this anomaly. This can be accommodated in the SCFT if the original $\operatorname{Sp}(n)$ also has the same anomaly. This again agrees with the expectation from the ADHM construction.

Both $G_{2}$ and $F_{4}$ cannot have an anomaly. However, they break on the Higgs branch to groups that can, $\mathrm{SU}(2)$ for $G_{2}$ and $\mathrm{Sp}(3)$ for $F_{4}$. Therefore for these to be possible the anomaly must vanish on the Higgs branch. This is true for $G_{2}$ as the $\mathbf{4}$ of $\mathrm{SU}(2)$ does not contribute to the anomaly. However, this is not true for $F_{4}$ as the $\mathbf{1 4}^{\prime}$ of $\mathrm{Sp}(3)$ does contribute to the anomaly. Thus this excludes $F_{4}$, since global anomaly associated to $\pi_{4}(G)$ cannot be canceled by adding topological degrees of freedom; see e.g. section 5 of [15]. The existence of the $G_{2}$ theory is still an open question.

\section{Two-dimensional theories}

In this section we analyze the $2 \mathrm{~d} \mathcal{N}=(0,4)$ theories. We denote the R-symmetry as $\mathrm{SU}(2)_{R} \times \mathrm{SU}(2)_{I}$ and the general form of the anomaly polynomial is written as

$$
I_{4}^{\text {full }}=-n_{v} c_{2}(R)+d_{H} c_{2}(I)+\frac{2 d_{H}-n_{f}}{24} p_{1}(T)+\frac{k_{G}}{4} \operatorname{Tr}\left(F_{G}^{2}\right),
$$

where $n_{V}, d_{H}, n_{F}$ and $k_{G}$ are the unknown coefficients determined below. Note that the $\mathrm{SU}(2)_{I}$ and the gravitational part of the anomaly can be matched directly on the Higgs branch. We also note that there are no global gauge anomalies in $2 \mathrm{~d}$ since $\pi_{2}(G)=0$ for all Lie groups.

In $2 \mathrm{~d}$, we can consider the slightly generalized situation: we can also have Fermi multiplets in addition to hypermultiplets on a generic point of the Higgs branch. Fermi multiplet consists of a single left-moving Weyl fermion transforming some representation $R_{F}$ under $G .{ }^{4}$ In this section, we also examine how the anomaly matching changes when we allow the Fermi multiplets as the massless spectrum. ${ }^{5}$

\section{1 $G$ is of type Sp or one of the exceptionals}

In this case, the unbroken subgroup $G^{\prime}$ is simple. If we denote the representations of Fermi multiplets under $G^{\prime}$ as $\sum_{m} \mathbf{N}_{\mathbf{m}}$, then the anomaly polynomial of free multiplets is given as

$$
I_{4}^{\text {free }}=c_{2}(D)+\frac{2+d_{R^{\prime}}}{2} c_{2}(I)+\frac{2+d_{R^{\prime}}-\sum_{m} N_{m}}{24} p_{1}(T)+\frac{2 T^{G^{\prime}}\left(R^{\prime}\right)-2 \sum_{m} T^{G^{\prime}}\left(\mathbf{N}_{\mathbf{m}}\right)}{4} \operatorname{Tr}\left(F_{G^{\prime}}^{2}\right) .
$$

On the other hand, by using (A.2) and (A.3), the anomaly (6.1) becomes

$$
I_{4}^{\text {generic }}=\left(k_{G}-n_{v}\right) c_{2}(D)+d_{H} c_{2}(I)+\frac{2 d_{H}-n_{f}}{24} p_{1}(T)+\frac{m k_{G}}{4} \operatorname{Tr}\left(F_{G^{\prime}}^{2}\right),
$$

where $m$ is 3 for $G_{2}$ and 1 for other cases.

\footnotetext{
${ }^{4}$ Some references (e.g. [25]) define a pair of left-moving Weyl fermions transforming in conjugate representations as a $2 \mathrm{~d} \mathcal{N}=(0,4)$ Fermi multiplet. Here we choose not to use such a definition.

${ }^{5}$ In this note, we only consider Fermi multiplets transforming non-trivially under $G^{\prime}$. The effect of neutral Fermi multiplets is to change the value of the gravitational anomaly.
} 
Without Fermi multiplets. If we assume that there are no Fermi multiplets, the anomalies (6.2) and (6.3) can be matched by the data summarized in table 3.

The cases with $G=E_{8}, E_{7}, E_{6}, F_{4}$ reproduce the anomaly on a single self-dual string 6 in minimal $6 \mathrm{~d} \mathcal{N}=(1,0)$ theories for $n=12,8,7,5$ :

$$
I_{4}^{\text {string }}=-(n-1) c_{2}(R)+(3 n-7) c_{2}(I)+\frac{3 n-7}{12} p_{1}(T)+\frac{n}{4} \operatorname{Tr} F_{G}^{2} .
$$

The case with $G=\operatorname{Sp}(n)$ reproduces the anomaly of $O(1) \times \operatorname{Sp}(n)$ half-hypers as in $4 \mathrm{~d}$ and $6 \mathrm{~d}$. To the best of our knowledge, we do not know an example of $2 \mathrm{~d} \mathcal{N}=(0,4)$ SCFT with Higgs branch $M_{G_{2}}$ and no Fermi multiplets.

With Fermi multiplets. Next we consider the cases with Fermi multiplets on the Higgs branch.

As examples, let us consider $n_{f}$ fundamental Fermi multiplets of $G^{\prime}$. For the $G=E_{7}$, the anomaly is given by

$$
I_{4}^{\text {full }}=-\left(7-n_{f}\right) c_{2}(R)+17 c_{2}(I)+\frac{17-3 n_{f}}{12} p_{1}(T)+\frac{8-n_{f}}{4} \operatorname{Tr}\left(F_{E_{7}}^{2}\right)+\frac{1}{4} \operatorname{Tr}\left(F_{\mathrm{SO}\left(n_{f}\right)}^{2}\right),
$$

where we included the $\mathrm{SO}\left(n_{f}\right)$ symmetry acting on Fermi multiplets. This anomaly precisely agrees with that of a single string in $6 \mathrm{~d} E_{7}$ gauge theory with $n_{f} / 2$ hypermultplets. Similarly, $G=E_{6}, F_{4}$ cases reproduce the anomaly of a single string in $6 \mathrm{~d} G=E_{6}, F_{4}$ gauge theory with $n_{f}$ fundamental hypermultiplets.

Finally, we consider $G=G_{2}$. The anomaly can be matched by

$$
I_{4}^{\text {full }}=-\frac{7-n_{f}}{3} c_{2}(R)+3 c_{2}(I)+\frac{3-n_{f}}{12} p_{1}(T)+\frac{10-n_{f}}{12} \operatorname{Tr}\left(F_{G_{2}}^{2}\right)+\frac{1}{4} \operatorname{Tr}\left(F_{\mathrm{SU}\left(n_{f}\right)}^{2}\right),
$$

where we included the $\mathrm{SU}\left(n_{f}\right)$ flavor symmetry acting on the Fermi multiplets. For $n_{f}=$ $1,4,7,(6.6)$ reproduces the anomaly of a string in the $6 \mathrm{~d} G_{2}$ gauge theory with $n_{f}=1,4,7$ fundamental hypermultiplets.

\section{2 $G$ is of type SO}

In this case, the unbroken group is $\mathrm{SU}(2)_{F} \times \mathrm{SO}(n-4)$. If we denote the representation of the Fermi multiplets by $\sum_{m}\left(\mathbf{n}_{\mathbf{m}}, \mathbf{N}_{\mathbf{m}}\right)$, the anomaly of the free multiplets is given by

$$
\begin{aligned}
I_{4}^{\text {free }}= & c_{2}(D)+(n-3) c_{2}(I)+\frac{2 n-6-\sum_{m} n_{m} N_{m}}{24} p_{1}(T) \\
& +\frac{n-4-2 \sum_{m} N_{m} T^{\mathrm{SU}(2)_{F}}\left(\mathbf{n}_{\mathbf{m}}\right)}{4} \operatorname{Tr}\left(F_{F}^{2}\right)+\frac{2-\sum_{m} n_{m} T^{\mathrm{SO}(n-4)}\left(\mathbf{N}_{\mathbf{m}}\right)}{2} \operatorname{Tr}\left(F_{\mathrm{SO}(n-4)}^{2}\right) .
\end{aligned}
$$

On the other hand, by using (A.5), the anomaly (6.1) becomes

$$
I_{4}^{\text {generic }}=\left(k_{G}-n_{v}\right) c_{2}(D)+d_{H} c_{2}(I)+\frac{2 d_{H}-n_{F}}{24} p_{1}(T)+\frac{k_{G}}{4} \operatorname{Tr}\left(F_{F}^{2}\right)+\frac{k_{G}}{4} \operatorname{Tr}\left(F_{\mathrm{SO}(n-4)}^{2}\right) .
$$

\footnotetext{
${ }^{6}$ We have subtracted the anomaly of the center-of-mass mode from the result presented in [18].
} 
Without Fermi multiplets. Comparing (6.7) and (6.8) in the case of Fermi multiplets, the anomaly can be solved by

$$
n_{v}=3, \quad d_{H}=n-3, \quad k_{G}=4,
$$

if we ignore the $\mathrm{SU}(2)_{F}$ part. This precisely agrees with the values of the $\mathrm{SU}(2)$ gauge theory with $n$ half-hypers, though it is anomalous in 2 d. If we include the matching of $\mathrm{SU}(2)_{F}$, the solution exists only for $G=\mathrm{SO}(8)$ and we obtain the anomaly of (6.4) for $n=4$. Indeed, the worldsheet theory on a single string in minimal $6 \mathrm{~d} \mathcal{N}=(1,0) \mathrm{SCFT}$ for $n=4$ has the Higgs branch $M_{\mathrm{SO}(8)}$.

With Fermi multiplets. Let us consider the cases with Fermi multiplets. The matching of $\mathrm{SU}(2)_{F}$ puts a constraint

$$
n-4-2 \sum_{m} N_{m} T^{\mathrm{SU}(2)_{F}}\left(\mathbf{n}_{\mathbf{m}}\right)=4-2 \sum_{m} n_{m} T^{\mathrm{SO}(n-4)}\left(\mathbf{N}_{\mathbf{m}}\right) .
$$

An example of solution of these constraints is obtained by setting $1 \leq m \leq n-8, \mathbf{N}_{\mathbf{m}}=\mathbf{1}$ and $\mathbf{n}_{\mathbf{m}}=\mathbf{2}$ for all $m$. The anomaly polynomial is

$$
I_{4}^{\text {full }}=-3 c_{2}(R)+(n-3) c_{2}(I)+\frac{5}{12} p_{1}(T)+\operatorname{Tr}\left(F_{\mathrm{SO}(n)}^{2}\right)+\frac{1}{4} \operatorname{Tr}\left(F_{\mathrm{Sp}(n-8)}^{2}\right),
$$

where we have included the global symmetry acting on $(n-8)$ free Fermi multiplets. This is precisely the anomaly of a single string in $6 \mathrm{~d} \mathrm{SO}(n)$ gauge theory with $(n-8)$ fundamental hypermultiplets.

\section{3 $\quad G$ is of type $\mathrm{SU}$}

If we denote the representation of the Fermi multiplets as $\oplus_{m}\left(\mathbf{N}_{\mathbf{m}}\right)_{\mathbf{n}_{\mathbf{m}}}$ under $\mathrm{SU}(n-2) \times$ $\mathrm{U}(1)_{F}$, the anomaly of the free multiplets is

$$
\begin{aligned}
I_{4}^{\text {free }}= & c_{2}(D)+(n-1) c_{2}(I)+\frac{2 n-2-\sum_{m} N_{m}}{24} p_{1}(T)+\frac{2-2 \sum_{m} T^{\mathrm{SU}(n-2)}\left(\mathbf{N}_{\mathbf{m}}\right)}{4} \operatorname{Tr}\left(F_{\mathrm{SU}(n-2)}^{2}\right) \\
& -\left(n^{2}(n-2)-\frac{1}{2} \sum_{m} N_{m} n_{m}^{2}\right) c_{1}\left(\mathrm{U}(1)_{F}\right)^{2} .
\end{aligned}
$$

On the other hand, by using the decomposition (A.7), we have the anomaly

$$
\begin{aligned}
I_{4}^{\text {generic }}= & \left(k_{\mathrm{SU}(n)}-n_{v}\right) c_{2}(D)+d_{H} c_{2}(I)+\frac{2 d_{H}-\sum_{m} N_{m}}{24} p_{1}(T)+\frac{k_{\mathrm{SU}(n)}}{4} \operatorname{Tr}\left(F_{\mathrm{SU}(n-2)}^{2}\right) \\
& -k_{\mathrm{SU}(n)} n(n-2) c_{1}\left(\mathrm{U}(1)_{F}\right)^{2} .
\end{aligned}
$$

Without Fermi multiplets. Let us first consider the case $n \geq 4$. If we ignore the $\mathrm{U}(1)_{F}$ part, the matching between (6.12) and (6.13) can be solved by

$$
n_{v}=1, \quad d_{H}=n-1, \quad k_{\mathrm{SU}(n)}=2
$$


which precisely agrees with the values of the U(1) gauge theory with $n$ hypermultiplets, though it is anomalous in $2 \mathrm{~d}$. The matching of $\mathrm{U}(1)_{F}$ forces us to set $n=2$, which contradicts with our assumption.

When $G=\mathrm{SU}(2)$, the matching can be solved by

$$
I_{4}^{\text {full }}=-\left(k_{\mathrm{SU}(2)}-1\right) c_{2}(R)+c_{2}(I)+\frac{1}{12} p_{1}(T)+\frac{k_{\mathrm{SU}(2)}}{4} \operatorname{Tr}\left(F_{\mathrm{SU}(2)}^{2}\right),
$$

where $k_{\mathrm{SU}(2)}$ is an undetermined coefficient. If we set $k_{\mathrm{SU}(2)}=1$, we reproduce the anomaly of the $\mathrm{O}(1) \times \mathrm{SU}(2)$ half-hyper as in $4 \mathrm{~d}$ and $6 \mathrm{~d}$.

When $G=\mathrm{SU}(3)$, the matching can be solved by

$$
I_{4}^{\text {full }}=-2 c_{2}(R)+2 c_{2}(I)+\frac{1}{6} p_{1}(T)+\frac{3}{4} \operatorname{Tr}\left(F_{\mathrm{SU}(3)}^{2}\right),
$$

which coincides with the anomaly (6.4) for $n=3$. Indeed, the worldsheet theory of a single string in minimal $6 \mathrm{~d} \mathcal{N}=(1,0)$ SCFT for $n=3$ is the SCFT with Higgs branch $M_{\mathrm{SU}(3)}$.

With Fermi multiplets. Let us consider the case with Fermi multiplets for $n \geq 4$. We consider two cases. When $\mathbf{N}_{\mathbf{m} \geq \mathbf{1}}=\mathbf{1}$, the matching can be solved by

$$
n_{V}=1, \quad d_{H}=n-1, \quad k_{\mathrm{SU}(n)}=2
$$

as long as the $\mathrm{U}(1)_{F}$ charges satisfy

$$
2 n(n-2)=n^{2}(n-2)-\frac{1}{2} \sum_{m} n_{m}^{2} .
$$

An example of solutions of (6.18) is obtained by setting $1 \leq m \leq 2 n$ and $n_{m}=(n-2)$ for all $m$. The full anomaly is

$$
I_{4}^{\text {full }}=-c_{2}(R)+(n-1) c_{2}(I)-\frac{1}{12} p_{1}(T)+\frac{2}{4} \operatorname{Tr}\left(F_{\mathrm{SU}(n)}^{2}\right)+\frac{1}{4} \operatorname{Tr}\left(F_{\mathrm{SU}(2 n)_{F}}^{2}\right),
$$

where we have included the contribution of the flavor symmetry $\mathrm{SU}(2 n)_{F}$, acting on the Fermi multiplets of the same $\mathrm{U}(1)_{F}$ charges. This is precisely the anomaly of a single string in $6 \mathrm{~d} \mathrm{SU}(n)$ gauge theory with $2 n$ fundamental hypermultiplets.

When $\mathbf{N}_{\mathbf{1}}=(\mathbf{n}-\mathbf{2}), \mathbf{N}_{\mathbf{m} \geq \mathbf{2}}=\mathbf{1}$, the matching can be solved by

$$
n_{v}=0, \quad d_{H}=n-1, \quad k_{\mathrm{SU}(n)}=1
$$

as long as the $\mathrm{U}(1)_{F}$ charges satisfy

$$
n(n-2)=n^{2}(n-2)-\frac{(n-2) n_{1}^{2}}{2}-\frac{1}{2} \sum_{m \geq 2} n_{m}^{2} .
$$

An example of solutions of (6.21) is obtained by setting $1 \leq m \leq n+9, n_{1}=(n-4)$ and $n_{m}=(n-2)$ for all $m \geq 2$. The total anomaly is given by

$$
I_{4}^{\text {full }}=(n-1) c_{2}(I)-\frac{1}{3} p_{1}(T)+\frac{1}{4} \operatorname{Tr}\left(F_{\mathrm{SU}(n)}^{2}\right)+\frac{1}{4} \operatorname{Tr}\left(F_{\mathrm{SU}(n+8)_{F}}^{2}\right),
$$

where we have included the global symmetry $\mathrm{SU}(n+8)$ acting on the Fermi multiplets of the same $\mathrm{U}(1)_{F}$ charge. This precisely agrees with the anomaly of a single string in $6 \mathrm{~d}$ $\mathrm{SU}(n)$ gauge theory with $N_{f}=n+8, N_{\Lambda^{2}}=1$ hypermultiplets. 


\section{Acknowledgments}

The authors would like to thank Kantaro Ohmori and Kazuya Yonekura for useful discussions. HS is partially supported by the Programs for Leading Graduate Schools, MEXT, Japan, via the Leading Graduate Course for Frontiers of Mathematical Sciences and Physics. HS is also supported by JSPS Research Fellowship for Young Scientists. The work of YT is partially supported in part by JSPS Grant-in-Aid for Scientific Research No. 25870159. The work of YT and GZ are partially supported by WPI Initiative, MEXT, Japan at IPMU, the University of Tokyo.

\section{A Decomposition of characteristic classes}

In this appendix, we collect the formulas relating the characteristic classes for $G$ and $G^{\prime}$, used in the main body of the paper. We define the Tr by the trace in the adjoint representation, divided by the dual Coxeter number of $G$. The Dynkin index of the representation $R$ of gauge group $G$ relates the $\operatorname{Tr} F_{G}^{2}$ via

$$
\operatorname{tr}_{R} F_{G}^{2}=T^{G}(R) \operatorname{Tr} F_{G}^{2},
$$

where $\operatorname{tr}_{R}$ is the trace in the representation $R$. We list the values of $T^{G}(R)$ relevant in this paper in table 4 .

When $G$ is one of the exceptionals. Since there are no independent quartic Casimir invariants in this case, we only have to consider $\operatorname{Tr} F_{G}^{2}$. The unbroken subgroup $G^{\prime}$ is simple. The formula is

$$
\operatorname{Tr}\left(F_{G}^{2}\right)=4 c_{2}(D)+m \operatorname{Tr} F_{G^{\prime}}^{2},
$$

where $m=3$ for $G_{2}$ and 1 for any other group.

When $\boldsymbol{G}$ is of type Sp. The unbroken subgroup is $\operatorname{Sp}(n-1)$ in this case. The $\operatorname{Tr} F_{\mathrm{Sp}(n)}^{2}$ is related that of $\operatorname{Sp}(n-1)$ via

$$
\operatorname{Tr}\left(F_{\mathrm{Sp}(n)}^{2}\right)=4 c_{2}(D)+\operatorname{Tr} F_{\mathrm{Sp}(n-1)}^{2} .
$$

The $\operatorname{tr}_{\text {fund }} F_{\mathrm{Sp}(n)}^{4}$ is related by

$$
\operatorname{tr}_{\text {fund }} F_{\mathrm{Sp}(n)}^{4}=2 c_{2}(D)^{2}+\operatorname{tr}_{\text {fund }} F_{\mathrm{Sp}(n-1)}^{4} .
$$

When $G$ is of type SO. The unbroken subgroup is $\mathrm{SU}(2)_{F} \times \mathrm{SO}(n-4)$ in this case. The $\operatorname{Tr} F_{\mathrm{SO}(n)}^{2}$ is related via

$$
\operatorname{Tr} F_{\mathrm{SO}(n)}^{2}=4 c_{2}(D)+\operatorname{Tr} F_{F}^{2}+\operatorname{Tr} F_{\mathrm{SO}(n-4)}^{2} .
$$

The $\operatorname{tr}_{\text {fund }} F_{\mathrm{SO}(n)}^{4}$ is related by

$$
\operatorname{tr}_{\text {fund }} F_{\mathrm{SO}(n)}^{4}=4 c_{2}(D)^{2}+2 \operatorname{tr}_{\text {fund }} F_{F}^{4}+12 c_{2}(D) \operatorname{tr}_{\text {fund }} F_{\mathrm{SO}(n-4)}^{2}+\operatorname{tr}_{\text {fund }} F_{\mathrm{SO}(n-4)}^{4} \text {. }
$$


When $G$ is of type SU. First, we assume $n \geq 4$. The $\operatorname{Tr} F_{\mathrm{SU}(n)}^{2}$ becomes

$$
\operatorname{Tr} F_{\mathrm{SU}(n)}^{2}=4 c_{2}(D)-4 n(n-2) c_{1}\left(\mathrm{U}(1)_{F}\right)^{2}+\operatorname{Tr}\left(F_{\mathrm{SU}(n-2)}^{2}\right),
$$

and $\operatorname{tr}_{\text {fund }} F_{\mathrm{SU}(n)}^{4}$ becomes

$$
\begin{aligned}
\operatorname{tr}_{\text {fund }} F_{\mathrm{SU}(n)}^{4}= & \operatorname{tr}_{\text {fund }} F_{\mathrm{SU}(n-2)}^{4}-8 c_{1}\left(\mathrm{U}(1)_{F}\right) \operatorname{tr}_{\text {fund }} F_{\mathrm{SU}(n-2)}^{3}-24 c_{1}\left(\mathrm{U}(1)_{F}\right)^{2} \operatorname{tr}_{\text {fund }} F_{\mathrm{SU}(n-2)}^{2} \\
& +2 c_{2}(D)^{2}-12(n-2)^{2} c_{1}\left(\mathrm{U}(1)_{F}\right)^{2} c_{2}(D)+2 n(n-2)\left(n^{2}-6 n+12\right) c_{1}\left(\mathrm{U}(1)_{F}\right)^{4}
\end{aligned}
$$

The cases of $\mathrm{SU}(2)$ and $\mathrm{SU}(3)$ are quite exceptional since these groups have no independent quartic Casimir and we only have to consider $\operatorname{Tr} F^{2}$. For $G=\mathrm{SU}(2), \mathrm{SU}(2)_{R}$ is identified with the original $G$ and we simply take

$$
\operatorname{Tr} F_{\mathrm{SU}(2)}^{2}=4 c_{2}(D)=4 c_{2}(R) .
$$

For the case of SU(3), we use

$$
\operatorname{Tr} F_{\mathrm{SU}(3)}^{2}=4 c_{2}(D)-12 c_{1}\left(\mathrm{U}(1)_{F}\right)^{2} .
$$

Open Access. This article is distributed under the terms of the Creative Commons Attribution License (CC-BY 4.0), which permits any use, distribution and reproduction in any medium, provided the original author(s) and source are credited.

\section{References}

[1] M.F. Atiyah, N.J. Hitchin, V.G. Drinfeld and Y.I. Manin, Construction of Instantons, Phys. Lett. A 65 (1978) 185 [inSPIRE].

[2] E. Witten, Small instantons in string theory, Nucl. Phys. B 460 (1996) 541 [hep-th/9511030] [INSPIRE].

[3] M.R. Douglas and G.W. Moore, D-branes, quivers and ALE instantons, hep-th/9603167 [INSPIRE].

[4] J.A. Minahan and D. Nemeschansky, An $N=2$ superconformal fixed point with $E_{6}$ global symmetry, Nucl. Phys. B 482 (1996) 142 [hep-th/9608047] [INSPIRE].

[5] J.A. Minahan and D. Nemeschansky, Superconformal fixed points with $E_{n}$ global symmetry, Nucl. Phys. B 489 (1997) 24 [hep-th/9610076] [InSPIRE].

[6] O.J. Ganor and A. Hanany, Small $E_{8}$ instantons and tensionless noncritical strings, Nucl. Phys. B 474 (1996) 122 [hep-th/9602120] [INSPIRE].

[7] N. Seiberg and E. Witten, Comments on string dynamics in six-dimensions, Nucl. Phys. B 471 (1996) 121 [hep-th/9603003] [INSPIRE].

[8] O. Aharony and Y. Tachikawa, A Holographic computation of the central charges of $D=4$, $N=2$ SCFTs, JHEP 01 (2008) 037 [arXiv:0711.4532] [INSPIRE].

[9] K. Ohmori, H. Shimizu and Y. Tachikawa, Anomaly polynomial of E-string theories, JHEP 08 (2014) 002 [arXiv: 1404.3887] [INSPIRE].

[10] A.D. Shapere and Y. Tachikawa, Central charges of $N=2$ superconformal field theories in four dimensions, JHEP 09 (2008) 109 [arXiv:0804.1957] [INSPIRE]. 
[11] K. Ohmori, H. Shimizu, Y. Tachikawa and K. Yonekura, Anomaly polynomial of general $6 d$ SCFTs, Prog. Theor. Exp. Phys. 2014 (2014) 103B07 [arXiv: 1408.5572] [InSPIRE].

[12] K. Intriligator, $6 d, \mathcal{N}=(1,0)$ Coulomb branch anomaly matching, JHEP 10 (2014) 162 [arXiv: 1408.6745] [INSPIRE].

[13] C. Beem, M. Lemos, P. Liendo, W. Peelaers, L. Rastelli and B.C. van Rees, Infinite Chiral Symmetry in Four Dimensions, Commun. Math. Phys. 336 (2015) 1359 [arXiv:1312.5344] [INSPIRE].

[14] D. Gaiotto, A. Neitzke and Y. Tachikawa, Argyres-Seiberg duality and the Higgs branch, Commun. Math. Phys. 294 (2010) 389 [arXiv:0810.4541] [INSPIRE].

[15] I. García-Etxebarria, H. Hayashi, K. Ohmori, Y. Tachikawa and K. Yonekura, $8 d$ gauge anomalies and the topological Green-Schwarz mechanism, JHEP 11 (2017) 177 [arXiv: 1710.04218] [INSPIRE].

[16] M. Lemos and P. Liendo, $\mathcal{N}=2$ central charge bounds from $2 d$ chiral algebras, JHEP 04 (2016) 004 [arXiv: 1511.07449] [INSPIRE].

[17] H.-C. Kim, S. Kim and J. Park, 6d strings from new chiral gauge theories, arXiv: 1608.03919 [INSPIRE].

[18] H. Shimizu and Y. Tachikawa, Anomaly of strings of $6 d \mathcal{N}=(1,0)$ theories, JHEP 11 (2016) 165 [arXiv: 1608. 05894] [INSPIRE].

[19] M. Del Zotto and G. Lockhart, On Exceptional Instanton Strings, JHEP 09 (2017) 081 [arXiv: 1609.00310] [INSPIRE].

[20] M. Bershadsky and C. Vafa, Global anomalies and geometric engineering of critical theories in six-dimensions, hep-th/9703167 [INSPIRE].

[21] S.M. Kuzenko and S. Theisen, Correlation Functions of Conserved Currents in $\mathcal{N}=2$ Superconformal Theory, Class. Quant. Grav. 17 (2000) 665 [hep-th/9907107] [INSPIRE].

[22] P.C. Argyres, K. Maruyoshi and Y. Tachikawa, Quantum Higgs branches of isolated $N=2$ superconformal field theories, JHEP 10 (2012) 054 [arXiv: 1206.4700] [INSPIRE].

[23] E. Witten, An SU(2) Anomaly, Phys. Lett. B 117 (1982) 324 [inSPIRE].

[24] S. Elitzur and V.P. Nair, Nonperturbative Anomalies in Higher Dimensions, Nucl. Phys. B 243 (1984) 205 [InSPIRE].

[25] D. Tong, The holographic dual of $A d S_{3} \times S^{3} \times S^{3} \times S^{1}$, JHEP 04 (2014) 193 [arXiv: 1402.5135] [INSPIRE]. 